\title{
Mickey Mouse's negative affect facing mistakes
}

\author{
Vassilissa Dolivo ${ }^{1}$
}

Published online: 1 April 2019

(C) The Psychonomic Society, Inc. 2019

\section{Summary}

Many of the scientists working in the field of 'animal behaviour' and especially of 'animal cognition' consider the most obvious factors for fitness maximization - for instance, nutritional reward maximization — as the sole motivators when a course of action must be chosen. Sweis, Thomas, and Redish (2018, PLOS Biology, 16(6), e2005853) show that even in a food-restricted environment in which it is vital to maximize food gaining, other factors, not obviously linked to fitness, play a role for decisionmaking - in the present case, avoidance of a negative affect linked to changing one's mind (a factor which does not improve foraging efficiency), and individual flavour preference (a factor which even impairs foraging efficiency).

Keywords Regret avoidance $\cdot$ Pondering strategy $\cdot$ Learning $\cdot$ Fitness $\cdot$ Rodents $\cdot$ Interindividual variability

Lunch time. Rush time. A food court with various queues and only 1 hour to eat. What would be your best strategy to maximize eating time while minimizing waiting time? As mentioned by Sweis, Thomas, and Redish (2018), people usually waste time in pondering before choosing a queue, although a better strategy would be to immediately choose a random queue and to decide afterwards whether to change for a quicker one. This last option nevertheless generates, when changing queue, an irrational negative feeling of 'having to abandon a partial investment', which will not appear with the first less efficient option. Sweis et al. show that after prolonged training in a foraging task, mice also choose to shift to a 'pondering' strategy, which protects them from regret, even though this switch in attitude plays no role in the quantity of food they manage to eat or in the energy they spend.

For 70 consecutive days, 31 mice had only 1 hour per day when they could eat by earning pellets of four different flavours in a variant of the Restaurant Row economic decisionmaking task. During this hour, each mouse had the opportunity to make laps in a square maze with four feeding sites. Each feeding site contained two distinct zones - an offer zone and a waiting zone - and delivered pellets with one specific flavour. Mice got acoustic information in the offer zone about the time they had to spend in the waiting zone before being

Vassilissa Dolivo

vassilissadolivo@hotmail.com

1 Behavioural Ecology, Institute for Ecology and Evolution Bern, Bern, Switzerland allowed to come back to the offer zone to receive the pellet. Mice could also choose to skip the waiting zone and directly go to the next feeding site, or to leave the waiting zone before the time stipulated by the acoustic signal. However in both situations, they did not get the pellet. xThe delay to each pellet was randomly selected for each trial between a range of offers. During the first 7 days, all offers lasted 1 second, but the range of possible offers escalated by steps. From Day 8 to Day 12, offers ranged from 1 second to 5 seconds, then from 1 second to 15 seconds, and finally from Day 18 until the end of the experiment (on Day 70), mice were given offers that ranged from 1 second to 30 seconds. Because mice had only 1 hour per day to get their food, the transition to an offer range of 1 to 30 seconds impaired them, until the moment they adapted their behaviour and modified their strategy. Thus, around Day 18, mice suffered a large drop in the number of pellets they managed to eat. By Day 32 , already, mice had adapted to the new environmental conditions and restored overall food intake. Among other adaptations, mice seemed to have learnt to quit the waiting zone more efficiently than by Day 18 (i.e. before excess time had been invested). Interestingly, with prolonged training, mice began to spend more time in the offer zone, presumably pondering whether to enter the waiting zone. Additionally, they entered the waiting zone less often, thus skipping 'time-expensive' offers, but they also reduced the frequency of leaving the waiting zone before the time needed to get the pellet. This did not improve the delivery efficiency of pellets, but allowed for a reduction of change-of-mind decisions. The authors argue that the reason for adopting this new strategy was to avoid regret. 
One interesting point in this study is that the authors have considered each individual's preference for specific flavours. Mice indeed have stable preferences, which vary between individuals. Taking into consideration individual tastes allowed the authors to demonstrate that skipping expensive offers was more difficult for preferred flavours. Although mice were restricted in food, they behaved economically less efficiently when having to decide what to do in preferred feeding sites. At first sight, from a Darwinian perspective and at the individual level, this may appear detrimental for each individual's fitness. But the fact that distinct preferences exist in a population could lead to various specializations in foraging, and thus decrease the competition when food is restricted. It therefore might increase the fitness at the population level. By considering only the average preference among individuals for one type of pellet, Sweis et al. (2018) would not have been able to reveal this essential result. Extrapolating a little further, we can conclude that variability, and even outliers, may sometimes be more enlightening than average group behaviour, which is too often privileged in many studies.

But let us come back to the main result. The switch to a 'pondering strategy' is not correlated with a change in the experimental task. It also does not increase mice's gain of pellets or minimize the energy they spent. Moreover, after prolonged training, a pause-and-look behaviour, termed 'vicarious trial and error', increased in the offer zone when choosing not to enter the waiting zone. 'Vicarious trial and error' behaviour (for definition, see Hu \& Amsel, 1995) is a well-studied mechanism associated with a representation of future outcomes and implies pondering over various decisions. Sweis et al. (2018) additionally mention other effects that they consider as being linked to regret, which were observed in the subsequent trials when mice were leaving the waiting zone before the time assigned to get the pellet, but not in the subsequent trials when mice were skipping the waiting zone. All these findings suggest that mice's behaviour is indeed triggered by regret avoidance.

Once again, and amazingly, behaviours triggered by regret avoidance do not obviously increase mice's fitness in Sweis et al.'s (2018) experiment, or the fitness of a person in a food court. Nevertheless, if pondering may be more costly than having to change one's mind in many circumstances, 'making a mistake only once' may be lethal in other situations. It may explain the emergence of regret avoidance from an evolutionary perspective. This nicely shows the complexity of interpreting the advantages or disadvantages of observed behaviours.

Studies about cooperation among rodents have shown that rats are able to help social partners according to direct reciprocity rules (Dolivo, Rutte, \& Taborsky, 2016). A rat will rather help another rat who previously helped him than a rat who never helped him before. Regarding Sweis's (2018) study, if rodents are sensitive to regrets, we can wonder whether they would also be sensitive to deception. In an experimental task allowing rats to provide partners with food, we could observe how test individuals would behave with co-operators switching to defectors. If rodents are indeed sensitive to deception, previous co-operators who stopped helping might receive even less help than defectors who never helped before.

As all mice certainly do not switch to a 'pondering strategy' at the same speed and rate, it could also be of great interest to investigate whether it is possible to find a correlation between individuals' trend to act in order to avoid regrets and other traits characterizing these individuals, such as their cognitive abilities in other learning tests, or their behavioural type. Behavioural types imply that individuals of a same species present distinct personality types regarding various traits (e.g., shier or bolder, more or less aggressive; Sih, Bell, \& Chadwick Johnson, 2004).

In analogy of Fourier's theorem in physics, where any periodic function can be decomposed as the sum of simple sine waves, observed behaviours could theoretically be explained by summing the effects of all mechanisms capable of influencing these behaviours. But most of these mechanisms frequently remain cryptic, and the motivators of these behaviours are not necessarily those assumed by the observers. We can conclude from Sweis et al.'s (2018) paper that when studying animal behaviour, we should always be aware that indirect benefits, not necessarily obvious to the experimenters and not necessarily related to reward maximization, very often also play a role in the decisions of individuals.

\section{References}

Dolivo, V., Rutte, C., \& Taborsky, M. (2016). Ultimate and proximate mechanisms of reciprocal altruism in rats. Learning \& Behavior, 44(3). doi:https://doi.org/10.3758/s13420-016-0236-Z

$\mathrm{Hu}, \mathrm{D} .$, \& Amsel, A. (1995). A simple test of the vicarious trial-and-error hypothesis of hippocampal function. Neurobiology, 92, 5506-5509.

Sih, A., Bell, A., \& Chadwick Johnson, J. (2004). Behavioral syndromes: An ecological and evolutionary overview. Trends in Ecology \& Evolution, 19(7). doi:https://doi.org/10.1016/j.tree.2004.04.009

Sweis, B. M., Thomas, M. J., \& Redish, A. D. (2018). Mice learn to avoid regret. PLOS Biology, 16(6), e2005853. doi:https://doi.org/10.1371/ journal.pbio. 2005853

Publisher's note Springer Nature remains neutral with regard to jurisdictional claims in published maps and institutional affiliations. 